Research Article

\title{
Uncertain Single-Machine Scheduling with Deterioration and Learning Effect
}

\author{
Jiayu Shen \\ Department of Public Basic Courses, Nanjing Institute of Industry Technology, Nanjing 210023, Jiangsu, China \\ Correspondence should be addressed to Jiayu Shen; fjcyue007@126.com
}

Received 3 February 2020; Accepted 6 April 2020; Published 7 May 2020

Academic Editor: Ada Che

Copyright (C) 2020 Jiayu Shen. This is an open access article distributed under the Creative Commons Attribution License, which permits unrestricted use, distribution, and reproduction in any medium, provided the original work is properly cited.

\begin{abstract}
A single-machine scheduling problem with deterioration and learning effect is studied in the present paper. The processing time and due date are considered uncertain variables due to lack of historical data. The aim is to minimize the makespan, total completion time, total weight completion time, and maximum lateness under an uncertain environment. To address the problem in an uncertain environment, the expected value model and pessimistic value model are developed. These models can be converted into equivalent models based on the inverse distribution method. It is proved that the corresponding dispatching rules can solve the problem optimally under different objective criteria. Finally, sensitivity analysis is used to illustrate the effectiveness of these rules.
\end{abstract}

\section{Introduction}

Single-machine scheduling is the basis of all scheduling problems, and many complex scheduling problems can be simplified into several single-machine problems. In recent years, many literature studies on scheduling are mainly concerned with the single scheduling problem. The traditional scheduling problem usually assumes that the processing time is a constant. This assumption may be impractical in many cases because the processing time may be prolonged or shortened as time passes. Some examples about deterioration or learning effect were mentioned in [1]. Raw material in the queue waiting to be processed deteriorates as time passes, which may lead to a certain loss (job deterioration). On an assembly line, workers become more skilled over time (learning effect). Many scholars had studied the scheduling problem with deterioration and learning effect [2-17].

Since environmental impact and resource constraint, emergencies can lead to uncertainty in processing time and due date. Many researchers studied the scheduling problems under stochastic or fuzzy environment. Optimal permutation policies in single-machine stochastic scheduling problems with learning effects were investigated [18]. A sequence-dependent single-machine scheduling problem with different learning effects was considered [19]. The processing times, setup times, and reliabilities/unreliabilities were considered as random variables. $\mathrm{Li}$ [20] studied a single-machine scheduling problem with random processing time and job-based learning effect recently. Ahmadizar and Hosseini [21, 22] considered single-machine scheduling problems with a position-based learning effect and fuzzy processing times. Toksari and Arık [23] considered singlemachine scheduling problems under position-dependent fuzzy learning effect with fuzzy processing times, and polynomially solvable algorithms for different confidence levels for these problems were proposed.

Most people believe that probability distribution is easy to obtain from the historical data, and then, we should use probability theory. However, the distribution function obtained in most practical problems is not close enough to the actual frequency. In this case, we have to invite the domain expert to assess the degree of belief that each event will occur. For example, a worker can only assess the processing time of a job based on his past experience. To address personal belief degrees, uncertainty theory was founded by Liu [24] in 2007 and refined it in 2010 [25]. Uncertainty theory is a branch of axiomatic mathematics. It has been developed in many fields such as uncertain calculus $[15,26-28]$, uncertain risk 
analysis [29-31], uncertain differential equation [32-35], and uncertain programming [36-40].

This paper studies the impacts of both deterioration and learning effect in a single-machine scheduling problem under an uncertain environment. The processing time and due date are considered uncertain variables. For example, in the rough machining operation process, due to machine failure, operation error, environmental impact, and many other uncertainties, workers usually evaluate the processing time according to their own experience. Much asymmetric information in the production and logistics process of the supply chain may lead to uncertainty and cause delays of the due date. It is more suitable for production practice to assume these parameters to be uncertain variables, which can ensure the feasibility and effectiveness of scheduling. The objective functions are makespan, total completion time, total weight completion time, and maximum lateness, respectively. Under different decision criteria, the expected value model and pessimistic value model are developed. Once uncertain distributions are given, the deterministic equivalence of the models is obtained by the uncertainty theory. To address the models effectively, corresponding dispatching rules are proposed.

The rest of the paper is organized as follows. To understand this paper better, basic definitions and results about uncertainty theory are introduced in Section 2. In Section 3, the problem under an uncertain environment is described and the mathematical models are constructed. In Section 4, to facilitate computation, the equivalence of the models is investigated and dispatching rules for the optimal solutions are presented. Section 5 gives an example of sensitivity analysis.

\section{Preliminary}

To describe an uncertain variable which refers to human uncertainty, Liu [24] established the uncertainty theory and it has been developed well up to now. Some necessary preliminary concepts about uncertainty theory are given.

Let $\Gamma$ be a nonempty set, $\mathscr{L}$ is a $\sigma$-algebra over $\Gamma$, and each element $\Lambda$ in $\mathscr{L}$ is called an event. A set function $M$ from $\mathscr{L}$ to $[0,1]$ is called an uncertain measure if it satisfies normality axiom, duality axiom, subadditivity axiom, and product axiom [24, 41].

The uncertain distribution $\Phi$ of an uncertain variable $\xi$ is defined by $\Phi(x)=M\{\xi \leq x\}$ for any real number $x$. The uncertain variables $\xi_{1}, \xi_{2}, \ldots, \xi_{m}$ are said to be independence (Liu [41]) if

$$
M\left\{\bigcap_{i=1}^{m}\left(\xi_{i} \in B_{i}\right)\right\}=\min _{1 \leq i \leq m} M\left\{\xi_{i} \in B_{i}\right\},
$$

for any Borel sets $B_{1}, B_{2}, \ldots, B_{n}$ of real numbers.

Uncertain measure has the following two useful properties: (i) for any events $\Lambda_{1} \subset \Lambda_{2}$, we have $M\left\{\Lambda_{1}\right\} \leq M\left\{\Lambda_{2}\right\}$, and (ii) for any events $\Lambda_{1}$ and $\Lambda_{2}$, we have

$$
M\left\{\Lambda_{1}\right\}+M\left\{\Lambda_{2}\right\}-1 \leq M\left\{\Lambda_{1} \cap \Lambda_{2}\right\} \leq M\left\{\Lambda_{1}\right\} \wedge M\left\{\Lambda_{2}\right\} .
$$

Definition 1 (see [24]). Let $\xi$ be an uncertain variable, and $\alpha \in(0,1]$. Then

$$
\xi_{\text {sup }}(\alpha)=\sup \{r \mid M\{\xi \geq r\} \geq \alpha\}
$$

is called the $\alpha$-optimistic value to $\xi$, and

$$
\xi_{\text {inf }}(\alpha)=\inf \{r \mid M\{\xi \leq r\} \geq \alpha\}
$$

is called the $\alpha$-pessimistic value to $\xi$.

Definition 2 (see [24]). An uncertain distribution $\Phi(x)$ is said to be regular if its inverse function $\Phi^{-1}(x)$ exists and is unique for each $\alpha \in(0,1)$. Then, the inverse function $\Phi^{-1}$ is called the inverse uncertainty distribution of $\xi$.

Theorem 1 (see [24]). Assume the constraint function $g\left(x, \xi_{1}, \xi_{2}, \ldots, \xi_{n}\right)$ is strictly increasing with respect to $\xi_{1}, \xi_{2}, \ldots, \xi_{k}$ and strictly decreasing with respect to $\xi_{k+1}, \xi_{k+2}, \ldots, \xi_{n}$. If $\xi_{1}, \xi_{2}, \ldots, \xi_{n}$ are independent uncertain variables with uncertainty distributions $\Phi_{1}, \Phi_{2}, \ldots, \Phi_{n}$, respectively, then the chance constraint

$$
\left.M\left\{g\left(x, \xi_{1}, \xi_{2}, \ldots, \xi_{n}\right) \leq 0\right)\right\} \geq \alpha,
$$

holds if and only if

$$
g\left(x, \Phi_{1}^{-1}(\alpha), \ldots, \Phi_{k}^{-1}(\alpha), \Phi_{k+1}^{-1}(1-\alpha), \ldots, \Phi_{n}^{-1}(1-\alpha)\right) \leq 0 .
$$

Theorem 2 (see [24]). Let $\xi$ be an uncertain variable. Then, the expected value of $\xi$ is defined by

$$
E[\xi]=\int_{0}^{+\infty} M\{\xi \geq r\} \mathrm{d} r-\int_{-\infty}^{0} M\{\xi \leq r\} \mathrm{d} r,
$$

provided that at least one of the two integrals is finite.

If $\xi$ and $\eta$ are independent uncertain variables, then

$$
E[a \xi+b \eta]=a E[\xi]+b E[\eta]
$$

holds for any real numbers $a$ and $b$.

\section{Mathematical Models}

The definition of the uncertain single-machine scheduling problem with deterioration and learning effect is given as follows.

Assume that $n$ independent and nonpreemptive jobs are processed on a single machine. All jobs are available at time 0 . Only one job can be processed at a time. The basic processing time of job $i$ is $\xi_{i}$, the due date of job $i$ is $d_{i}$, and the weight of job $i$ is $w_{i}$. The deterioration function $a(t)$ is an increasing function $(a(0) \geq 0)$ and is a differentiable convex function. We consider the similar actual processing time proposed by Lee [42]. The actual processing time of job $i$ is $\xi_{i}\left[a(t)+\beta r^{b}\right]$, where $b \leq 0$ is the learning coefficient and $t$ is the start processing time of job $i$.

The processing time, due date, and learning coefficient are assumed to be uncertain variables. In the real operating environment, the forgotten effect leads to job deterioration due to the frequent rotation of the task. With the increase of time and the accumulation of experience, workers become more skilled in operating the machine. Before constructing 
the mathematical model of the uncertain single-machine problem, the parameters for models are given as follows (Table 1 lists notations for the proposed models):

$$
\begin{aligned}
C_{\max } & =\max _{i=1,2, \ldots, n} C_{i}, \\
L_{\max } & =\max _{i=1,2, \ldots, n}\left\{C_{i}-d_{i}\right\} .
\end{aligned}
$$

Decision variables:

$x_{i, r}: \quad 1, \quad$ if job $i$ is processed at the $r$ th position, 0 , otherwise.

The relationship of variables is as follows:

$$
\begin{aligned}
p_{[r]} & =\sum_{i=1}^{n} x_{i, r} \cdot\left[\xi_{i}\left(a(t)+\beta r^{b}\right)\right], \quad r=1,2, \ldots, n, \\
C_{[r]} & =C_{[r-1]}+p_{[r]}, \quad r=1,2, \ldots, n ; j=1,2, \ldots, m, \\
C_{i} & =\sum_{r=1}^{n} x_{i, r} \cdot C_{[r]}, \quad i=1,2, \ldots, n .
\end{aligned}
$$

Equation (11) define the actual processing time of the $r$ th job on the machine. Clearly, the processing time changes with the position. Equation (12) define the completion time of the $r$ th job on the machine. Equation (13) reveal the relationship between the completion times.

When the processing time, due date, and learning coefficient are uncertain variables, how can we obtain an optimal solution in such an uncertain environment? First, we can use the uncertainty expectation to measure the uncertain variables. The expected value is the average of the uncertain variables on the uncertainty measure.

The problem is modeled under the expected value criterion:

$$
\begin{aligned}
& \min E\left[C_{\max }\right] \\
& \min E\left[\sum C_{i}\right] \\
& \min \quad E\left[\sum w_{i} C_{i}\right] \\
& \min E\left[L_{\text {max }}\right] \\
& \text { subject to } \sum_{i=1}^{n} x_{i, r}=1, \quad r=1,2, \ldots, n \\
& \sum_{r=1}^{n} x_{i, r}=1, \quad i=1,2, \ldots, n \\
& x_{i, r} \in\{0,1\}, \quad \forall i, r .
\end{aligned}
$$

This model minimizes the expected values of these objective functions subject to some constraints, respectively. Equation (14) ensure that each job can only be assigned to one position on the machine. Equation (14) ensure that each position can only process one job and there is no spare time on the machine. Constraint (14) gives the range of the decision variables.

In reality, the decision maker always tries to find the optimal solution within a small deviation. A preset target $\bar{f}$ is determined such that there exists a solution $x^{*}$ satisfying $M\{f(x) \leq \bar{f}\} \geq \alpha$. For example, let $\alpha=0.9$, the decision
TABLE 1: List of notations.

\begin{tabular}{lc}
\hline Notations & Definitions \\
\hline$i=1,2, \ldots, n$ & The index of job \\
$r=1,2, \ldots, n$ & The index of position \\
$\xi_{i}$ & Inherent processing time of job $i$ \\
$d_{i}$ & The due date of job $i$ \\
$w_{i}$ & The weight of job $i$ \\
$a(t)$ & Deterioration function \\
$p_{[r]}$ & The processing time of the $r$ th job on the machine \\
$b$ & Learning coefficient \\
$C_{\max }$ & The makespan \\
$C_{i}$ & The completion time of job $i$ \\
$C_{[r]}$ & The completion time of the $r$ th job on the machine \\
$\sum_{[} C_{i}$ & The total completion time \\
$\sum_{i} w_{i} C_{i}$ & The total weighted completion time \\
$L_{\max }$ & The maximum lateness \\
\hline
\end{tabular}

maker should determine a target $\bar{f}$ and then choose a solution $x$ which satisfies $M\{f(x) \leq \bar{f}\} \geq 0$.9. It means that if the decision maker chooses the solution $x$, the objective function $f(x)$ will be less than or equal to $\bar{f}$ at least $90 \%$. A pessimistic value model is conceived:

$$
\begin{array}{ll}
\min & \bar{f}_{1} \\
\min & \bar{f}_{2} \\
\min & \bar{f}_{3} \\
\min & \bar{f}_{4} \\
\text { subject to } & M\left\{C_{\max } \leq \bar{f}_{1}\right\} \geq \alpha_{1} \\
& M\left\{\sum C_{i} \leq \bar{f}_{2}\right\} \geq \alpha_{2} \\
& M\left\{\sum w_{i} C_{i} \leq \bar{f}_{3}\right\} \geq \alpha_{3} \\
& M\left\{L_{\max } \leq \bar{f}_{4}\right\} \geq \alpha_{4} \\
& \sum_{i=1}^{n} x_{i, r}=1, \quad r=1,2, \ldots, n \\
& \sum_{r=1}^{n} x_{i, r}=1, \quad i=1,2, \ldots, n \\
&
\end{array}
$$

where $\alpha_{i}, i=1,2, \ldots, 4$ are preset confidence levels.

This model is to calculate the pessimistic value to $f_{i}, i=1,2, \ldots, 4$. The detailed proof will be obtained in the next section. Constraint (15) ensure that the conditions hold under confidence levels $\alpha_{i}, i=1,2, \ldots, 4$, respectively.

\section{Equivalence of Uncertain Models}

It is noted that the above two models contain many uncertain variables, so it is difficult to solve them directly. In most uncertain programming literature studies, GA and PSO are used to find the approximate optimal solution. Although the effectiveness of these approaches is often illustrated by numerical experiments, the preciseness and generality of these approaches are not satisfactory. The uncertain models can be converted into deterministic forms with the help of uncertainty theory. 
Theorem 3. The expected value model can be converted into the following model equivalently:

$$
\begin{aligned}
& \min \max _{i=1,2, \ldots, n}\left\{\sum_{r=1}^{n} x_{i, r} \int_{0}^{1} \Phi_{C_{[r-1]}}^{-1}(\gamma) \mathrm{d} \gamma+\sum_{i=1}^{n} x_{i, r} \int_{0}^{1} \Phi_{\xi_{i}}^{-1}(\gamma)\left[a(t)+\beta r^{b}\right] \mathrm{d} \gamma\right\} \\
& \min \quad \sum_{i=1}^{n}\left\{\sum_{r=1}^{n} x_{i, r} \int_{0}^{1} \Phi_{C_{[r-1]}}^{-1}(\gamma) \mathrm{d} \gamma+\sum_{i=1}^{n} x_{i, r} \int_{0}^{1} \Phi_{\xi_{i}}^{-1}(\gamma)\left[a(t)+\beta r^{b}\right] \mathrm{d} \gamma\right\} \\
& \min \quad \sum_{i=1}^{n} w_{i}\left\{\sum_{r=1}^{n} x_{i, r} \int_{0}^{1} \Phi_{C_{[r-1]}^{-1}}(\gamma) \mathrm{d} \gamma+\sum_{i=1}^{n} x_{i, r} \int_{0}^{1} \Phi_{\xi_{i}}^{-1}(\gamma)\left[a(t)+\beta r^{b}\right] \mathrm{d} \gamma\right\} \\
& \min \max _{i=1,2, \ldots, n}\left\{\left\{\sum_{r=1}^{n} x_{i, r} \int_{0}^{1} \Phi_{C_{[r-1]}^{-1}}^{-1}(\gamma) \mathrm{d} \gamma+\sum_{i=1}^{n} x_{i, r} \int_{0}^{1} \Phi_{\xi_{i}}^{-1}(\gamma)\left[a(t)+\beta r^{b}\right] \mathrm{d} \gamma\right\}-\int_{0}^{1} \Phi_{d_{i}}^{-1}(\gamma) \mathrm{d} \gamma\right\} \\
& \text { subject to } \sum_{i=1}^{n} x_{i, r}=1, \quad r=1,2, \ldots, n \\
& \sum_{r=1}^{n} x_{i, r}=1, \quad i=1,2, \ldots, n \\
& x_{i, r} \in\{0,1\} \text {. }
\end{aligned}
$$

where $\Phi_{f}^{-1}$ represents the inverse uncertainty distribution of $f$.

$$
\begin{aligned}
E\left[C_{\max }\right] \geq \max _{i=1,2, \ldots, n} E\left[C_{i}\right] & =\max _{i=1,2, \ldots, n} E\left[\sum_{r=1}^{n} x_{i, r} C_{[r-1]}+\sum_{i=1}^{n} x_{i, r}\left[\xi_{i}\left(a(t)+\beta r^{b}\right)\right]\right] \\
& =\max _{i=1,2, \ldots, n}\left[\sum_{r=1}^{n} x_{i, r} E\left[C_{[r-1]}\right]+\sum_{i=1}^{n} x_{i, r} E\left[\xi_{i}\left(a(t)+\beta r^{b}\right)\right]\right] \\
& =\max _{i=1,2, \ldots, n}\left\{\sum_{r=1}^{n} x_{i, r} \int_{0}^{1} \Phi_{C_{[r-1]}^{-1}}(\gamma) \mathrm{d} \gamma+\sum_{i=1}^{n} x_{i, r} \int_{0}^{1} \Phi_{\xi_{i}}^{-1}(\gamma)\left[a(t)+\beta r^{b}\right] \mathrm{d} \gamma\right\} .
\end{aligned}
$$

Similarly, other objective functions can be obtained.

Theorem 4. The pessimistic value model can be converted into the following model equivalently:

$$
\begin{array}{ll}
\min & \Phi_{C_{\max }}^{-1}\left(\alpha_{1}\right) \\
\min & \sum^{-1} C_{i}\left(\alpha_{2}\right) \\
\min & \sum^{-1} w_{i} C_{i}\left(\alpha_{3}\right) \\
\min & \Phi_{L_{\max }^{-1}}\left(\alpha_{4}\right) \\
\text { subject to } & \sum_{i=1}^{n} x_{i, r}=1, \quad r=1,2, \ldots, n \\
& \sum_{r=1}^{n} x_{i, r}=1, \quad i=1,2, \ldots, n \\
&
\end{array}
$$

where $\alpha_{i}, i=1,2, \ldots, 4$ are preset confidence levels and $\Phi_{f}^{-1}$ represents the inverse uncertainty distribution of $f$.
Proof. Since the expectation operator is linear and according to Jensen's inequality, it yields 
$M\{\xi \leq \bar{f}\} \geq \alpha$ is equivalent to

$$
f\left(\Phi_{1}^{-1}(\alpha), \ldots, \Phi_{m}^{-1}(\alpha), \Phi_{m+1}^{-1}(1-\alpha), \ldots, \Phi_{n}^{-1}(1-\alpha)\right) \leq \bar{f},
$$

where $\alpha$ is a given confidence level.

According to the above conclusion, we can obtain the equivalent form of the pessimistic value model:

$$
\begin{array}{ll}
\min & \bar{f}_{1} \\
\min & \bar{f}_{2} \\
\min & \bar{f}_{3} \\
\min & \bar{f}_{4} \\
\operatorname{subject~to} & \Phi_{C_{\max }^{-1}}\left(\alpha_{1}\right) \leq \bar{f}_{1} \\
& \Phi_{\sum C_{i}}^{-1}\left(\alpha_{2}\right) \leq \bar{f}_{2} \\
& \Phi_{\sum w_{i} C_{i}}^{-1}\left(\alpha_{3}\right) \leq \bar{f}_{3} \\
& \Phi_{L_{\max }}^{-1}\left(\alpha_{4}\right) \leq \bar{f}_{4} \\
& \sum_{i=1}^{n} x_{i, r}=1, \quad r=1,2, \ldots, n \\
& \sum_{r=1}^{n} x_{i, r}=1, \quad i=1,2, \ldots, n
\end{array}
$$

where $\alpha_{i}, i=1,2, \ldots, 4$ are preset confidence levels and $\Phi_{f}^{-1}$ represents the inverse uncertainty distribution of $f$.

According to the definition of optimistic value (Definition 1), equation (23) is equivalent to equation (18), thus (23) are equivalent to equation (18), equations (23) are equivalent to equation (18), equations (23) are equivalent to equation (18). Thus, the theorem is proved.

Theorem 5. The optimal schedule of the makespan problem under the uncertain environment is obtained by the shortest processing time (SPT) rule.

Proof. An optimal schedule $\pi$ which consists of two adjacent jobs $\left(J_{i}\right.$ precedes $\left.J_{j}\right)$ is considered. Assume the start processing time of job $J_{i}$ is $A$. Let $C_{i}$ and $C_{j}$ represent the completion times of the jobs $J_{i}$ and $J_{j}$, respectively.

(1) Under the expectation value criterion $E\left[\xi_{i}\right]>E\left[\xi_{j}\right]$ :

$$
\begin{aligned}
E\left[C_{i}(\pi)\right]= & A+E\left[\xi_{i}\right]\left(a(A)+\beta r^{b}\right), \\
E\left[C_{j}(\pi)\right]= & A+E\left[\xi_{i}\right]\left(a(A)+\beta r^{b}\right) \\
& +E\left[\xi_{j}\right]\left[a\left(C_{i}(\pi)+\beta(r+1)^{b}\right)\right] .
\end{aligned}
$$

By exchanging the position of two jobs, a schedule $\pi^{\prime}$ is obtained:

$$
\begin{aligned}
E\left[C_{j}\left(\pi^{\prime}\right)\right]= & A+E\left[\xi_{j}\right]\left(a(A)+\beta r^{b}\right), \\
E\left[C_{i}\left(\pi^{\prime}\right)\right]= & A+E\left[\xi_{j}\right]\left(a(A)+\beta r^{b}\right) \\
& +E\left[\xi_{i}\right]\left[a\left(C_{j}\left(\pi^{\prime}\right)+\beta(r+1)^{b}\right)\right] .
\end{aligned}
$$

By comparing their sizes and according to Lagrange mean value theorem, it yields

$$
\begin{aligned}
E\left[C_{i}\left(\pi^{\prime}\right)\right]-E\left[C_{j}(\pi)\right]= & E\left[\xi_{j}\right]\left(a(A)+\beta r^{b}\right) \\
& +E\left[\xi_{i}\right]\left[a\left(C_{j}\left(\pi^{\prime}\right)+\beta(r+1)^{b}\right)\right] \\
& -E\left[\xi_{i}\right]\left(a(A)+\beta r^{b}\right) \\
& -E\left[\xi_{j}\right]\left[a\left(C_{i}(\pi)+\beta(r+1)^{b}\right)\right] \\
= & \beta\left(E\left[\xi_{j}\right]-E\left[\xi_{i}\right]\right)\left(r^{E[a]}-(r+1)^{b}\right) \\
& +E\left[\xi_{i}\right] a^{\prime}\left(\zeta_{1}\right) E\left[\xi_{j}\right]\left(a(A)+\beta r^{b}\right) \\
& -E\left[\xi_{j}\right] a^{\prime}\left(\zeta_{2}\right)\left[\xi_{i}\right]\left(a(A)+\beta r^{b}\right),
\end{aligned}
$$

where $A<\zeta_{1}<A+E\left[\xi_{j}\right]\left(a(A)+\beta r^{b}\right)$ and $A<\zeta_{2}$ $<A+E\left[\xi_{i}\right]\left(a(A)+\beta r^{b}\right)$.

Because $E\left[\xi_{i}\right]>E\left[\xi_{j}\right], r^{b} \geq(r+1)^{b}$, and $a^{\prime}\left(\zeta_{1}\right)<$ $a^{\prime}\left(\zeta_{2}\right)$, it yields $E\left[C_{i}\left(\pi^{\prime}\right)\right]<E\left[C_{j}(\pi)\right]$. It implies that the expected makespan under $\pi$ is smaller than that under $\pi^{\prime}$. This result contradicts the optimality of $\pi$.

(2) Under the pessimistic value criterion $M\left\{\xi_{i}>\xi_{j}\right\} \geq \alpha$, $r^{b} \geq(r+1)^{b}$, and $M\left\{a^{\prime}\left(\zeta_{1}\right)<a^{\prime}\left(\zeta_{2}\right)\right\} \geq \alpha$ :

$$
\begin{aligned}
& M\left\{C_{i}\left(\pi^{\prime}\right)-C_{j}(\pi)<0\right\} \\
& \quad=M\left\{\beta\left(\xi_{j}-\xi_{i}\right)\left[r^{a}-(r+1)^{a}\right]+\xi_{i} \alpha^{\prime}\left(\zeta_{1}\right) \xi_{j}\left(\alpha(A)+\beta r^{a}\right)-\xi_{j} \alpha^{\prime}\left(\zeta_{2}\right) \xi_{i}\left(\alpha(A)+\beta r^{a}\right)<0\right\} .
\end{aligned}
$$

Because $\quad\left\{C_{i}\left(\pi^{\prime}\right)-C_{j}(\pi)<0\right\} \supset\left\{\xi_{i}>\xi_{j}\right\} \cap\left\{r^{a} \geq\right.$ $\left.(r+1)^{a}\right\} \cap\left\{a^{\prime}\left(\zeta_{1}\right)<a^{\prime}\left(\zeta_{2}\right)\right\}$, and according to the two useful properties of uncertain measure, it yields

$$
\begin{aligned}
M\left\{C_{i}\left(\pi^{\prime}\right)-C_{j}(\pi)<0\right\} \geq & M\left\{\xi_{i}>\xi_{j}\right\}+M\left\{r^{b}-(r+1)^{b}\right\} \\
& +M\left\{a^{\prime}\left(\zeta_{1}\right)<a^{\prime}\left(\zeta_{2}\right)\right\}-2 \\
\geq & 3 \alpha-2 .
\end{aligned}
$$


It implies that the pessimistic value of makespan under $\pi$ is smaller than that under $\pi^{\prime}$. This result contradicts the optimality of $\pi$. Hence, the SPT rule gets the optimal solution. The theorem is proved.

Theorem 6. The optimal schedule of $\sum C_{i}$ problem under the uncertain environment is obtained by the SPT rule.

Proof. Theorem 5 implies that the start processing time of jobs after $J_{i}$ and $J_{j}$ can be advanced by changing the order. Therefore, the $\sum C_{i}$ in $\pi^{\prime}$ is less than that in $\pi$. This result contradicts the optimality of $\pi$. The theorem is proved.

Theorem 7. The optimal schedule of $\sum w_{i} C_{i}$ problem under the uncertain environment is obtained by the WSPT rule.

Proof. An optimal schedule $\pi$ which consists of two adjacent jobs $\left(J_{i}\right.$ precedes $\left.J_{j}\right)$ is considered, and the jobs are not arranged according to the WSPT rule. Hence, we have $\left(\xi_{i} / w_{i}\right)>\left(\xi_{j} / w_{j}\right)$.

(1) Under the expectation value criterion:

$$
\begin{aligned}
E\left[C_{i}(\pi)\right]= & A+E\left[\xi_{i}\right]\left(a(A)+\beta r^{b}\right), \\
E\left[C_{j}(\pi)\right]= & A+\beta E\left[\xi_{i}\right] r^{b}+E\left[\xi_{i}\right] a(A) \\
& +E\left[\xi_{j}\right]\left[a\left(C_{i}(\pi)+\beta E\left[\xi_{j}\right](r+1)^{b}\right)\right] .
\end{aligned}
$$

By exchanging the position of two jobs, a schedule $\pi^{\prime}$ is obtained:

$$
\begin{aligned}
E\left[C_{j}\left(\pi^{\prime}\right)\right]= & A+E\left[\xi_{j}\right]\left(a(A)+\beta r^{b}\right), \\
E\left[C_{i}\left(\pi^{\prime}\right)\right]= & A+\beta E\left[\xi_{j}\right] r^{b}+E\left[\xi_{j}\right] a(A) \\
& +E\left[\xi_{i}\right]\left[a\left(C_{j}\left(\pi^{\prime}\right)+\beta E\left[\xi_{i}\right](r+1)^{b}\right)\right] .
\end{aligned}
$$

Similar to Theorem 5 , it yields

$$
\begin{aligned}
\sum w_{i} E\left[C_{i}\left(\pi^{\prime}\right)\right]-\sum w_{j} E\left[C_{j}(\pi)\right] \\
=\beta\left(E\left[\xi_{j}\right]-E\left[\xi_{i}\right]\right)\left(r^{b}-(r+1)^{b}\right)\left(w_{i}+w_{j}\right)+\left(w_{i} E\left[\xi_{j}\right]-w_{j} E\left[\xi_{i}\right]\right)\left[a(A)+\beta(r+1)^{b}\right] \\
\quad+w_{i} E\left[\xi_{i}\right] a^{\prime}\left(\zeta_{1}\right) E\left[\xi_{j}\right]\left(a(A)+\beta r^{b}\right)-w_{j} E\left[\xi_{j}\right] a^{\prime}\left(\zeta_{2}\right) E\left[\xi_{i}\right]\left(a(A)+\beta r^{b}\right) .
\end{aligned}
$$

Because $E\left[\xi_{i}\right]>E\left[\xi_{j}\right], \quad r^{b} \geq(r+1)^{b}, \quad w_{j}>w_{i}$, $\left(E\left[\xi_{i}\right] / w_{i}\right)>\left(E\left[\xi_{j}\right] / w_{j}\right)$, and $a^{\prime}\left(\zeta_{1}\right)<a^{\prime}\left(\zeta_{2}\right)$, it yields $\sum w_{i} E\left[C_{i}\left(\pi^{\prime}\right)\right]<\sum w_{j} E\left[C_{j}(\pi)\right]$. It implies that the expected weighted sum under $\pi^{\prime}$ is smaller than that under $\pi$. This result contradicts the optimality of $\pi$.
(2) Under the pessimistic value criterion $M\left\{\xi_{i}>\xi_{j}\right\} \geq \alpha$, $w_{i}<w_{j}, \quad M\left\{r^{a} \geq(r+1)^{a}\right\} \geq \alpha, \quad$ and $\quad M\left\{a^{\prime}\left(\zeta_{1}\right)<\right.$ $\left.a^{\prime}\left(\zeta_{2}\right)\right\} \geq \alpha$.

Because $\left\{\xi_{i}>\xi_{j}\right\} \cap\left\{w_{i}<w_{j}\right\} \subset\left\{\left(\xi_{i} / w_{i}\right)>\left(\xi_{j} / w_{j}\right)\right\}$, it yields $M\left\{\left(\xi_{i} / w_{i}\right)>\left(\xi_{j} / w_{j}\right)\right\} \geq \alpha$ :

$$
\begin{aligned}
& M\left\{\sum w_{i} C_{i}\left(\pi^{\prime}\right)-\sum w_{j} C_{j}(\pi)<0\right\} \\
& =M\left\{\beta\left(\xi_{j}-\xi_{i}\right)\left(r^{b}-(r+1)^{b}\right)\left(w_{i}+w_{j}\right)+\left(w_{i} \xi_{j}-w_{j} \xi_{i}\right)\left[a(A)+\beta(r+1)^{b}\right]\right. \\
& \left.\quad+w_{i} \xi_{i} a^{\prime}\left(\zeta_{1}\right) \xi_{j}\left(a(A)+\beta r^{b}\right)-w_{j} \xi_{j} a^{\prime}\left(\zeta_{2}\right) \xi_{i}\left(a(A)+\beta r^{b}\right)<0\right\} .
\end{aligned}
$$

Because 


$$
\begin{aligned}
& \left\{\sum w_{i} C_{i}\left(\pi^{\prime}\right)-\sum w_{j} C_{j}(\pi)<0\right\}> \\
& \left\{\xi_{i}>\xi_{j}\right\} \cap\left\{r^{b} \geq(r+1)^{b}\right\} \\
& \cap\left\{\left(\xi_{i} / w_{i}\right)>\left(\xi_{j} / w_{j}\right)\right\} \cap a^{\prime}\left(\zeta_{1}\right)<a^{\prime}\left(\zeta_{2}\right),
\end{aligned}
$$

then according to the two useful properties of uncertain measure, it yields

$$
\begin{aligned}
M\left\{C_{i}\left(\pi^{\prime}\right)-C_{j}(\pi)<0\right\} \geq & M\left\{\xi_{i}>\xi_{j}\right\}+M\left\{r^{b} \geq(r+1)^{b}\right\} \\
& +M\left\{\xi_{i} / w_{i} n>q \xi_{j} / w_{j}\right\} \\
& +M\left\{a^{\prime}\left(\zeta_{1}\right)<a^{\prime}\left(\zeta_{2}\right)\right\}-3 \\
\geq & 4 \alpha-3 .
\end{aligned}
$$

It implies that the pessimistic value of $\sum w_{i} C_{i}$ under $\pi^{\prime}$ is smaller than that under $\pi$. This result contradicts the optimality of $\pi$. Hence, the SPT rule gets the optimal solution. The theorem is proved.

Theorem 8. The optimal schedule of $L_{\max }$ problem under uncertain environment is obtained by the EDD rule.

Proof. An optimal schedule $\pi$ which consists of two adjacent jobs $\left(J_{i}\right.$ precedes $\left.J_{j}\right)$ is considered, and the jobs are not arranged according to the EDD rule.

(1) Under the expectation value criterion, we have $E\left[d_{i}\right]>E\left[d_{j}\right]$. It implies that $E\left[\xi_{i}\right]>E\left[\xi_{j}\right]:$

$$
\begin{aligned}
E\left[L_{i}(\pi)\right]= & A+E\left[\xi_{i}\right] a(A)-E\left[d_{i}\right], \\
E\left[L_{j}(\pi)\right]= & A+\beta E\left[\xi_{i}\right] r^{b}+E\left[\xi_{i}\right] a(A) \\
& +E\left[\xi_{j}\right] a\left[A+\xi_{i}\left(a(A)+\beta r^{b}\right)\right] \\
& +\beta E\left[\xi_{j}\right](r+1)^{b}-E\left[d_{j}\right] .
\end{aligned}
$$

By exchanging the position of two jobs, a schedule $\pi^{\prime}$ is obtained:

$$
\begin{aligned}
E\left[L_{j}\left(\pi^{\prime}\right)\right]= & A+E\left[\xi_{j}\right] a(A)-E\left[d_{j}\right], \\
E\left[L_{i}\left(\pi^{\prime}\right)\right]= & A+\beta E\left[\xi_{j}\right] r^{b}+E\left[\xi_{j}\right] a(A) \\
& +E\left[\xi_{i}\right] a\left[A+\xi_{j}\left(a(A)+\beta r^{b}\right)\right] \\
& +\beta E\left[\xi_{i}\right](r+1)^{b}-E\left[d_{i}\right] .
\end{aligned}
$$

Because $E\left[d_{i}\right]>E\left[d_{j}\right]$ and $E\left[\xi_{i}\right]>E\left[\xi_{j}\right]$, it yields

$$
\max \left\{E\left[L_{i}\left(\pi^{\prime}\right)\right], E\left[L_{j}\left(\pi^{\prime}\right)\right]\right\} \leq \max \left\{E\left[L_{i}(\pi)\right], E\left[L_{j}(\pi)\right]\right\} .
$$

It implies that EDD is optimal.

(2) Under the pessimistic value criterion $M\left\{d_{i}>d_{j}\right\} \geq \alpha$. Because $\left\{d_{i}>d_{j}\right\} \subset\left\{\xi_{i}>\xi_{j}\right\}$, it yields $M\left\{\xi_{i}>\xi_{j}\right\} \geq \alpha$. And because

$$
\left\{\max \left\{L_{i}\left(\pi^{\prime}\right), L_{j}\left(\pi^{\prime}\right)\right\}-\max \left\{L_{i}(\pi), L_{j}(\pi)\right\}<0\right\} \supset\left\{d_{i}>d_{j}\right\} \cap\left\{\xi_{i}>\xi_{j}\right\},
$$

it yields

$$
\begin{aligned}
& M \\
& M \\
& \left.\quad \geq \max \left\{L_{i}\left(\pi^{\prime}\right), L_{j}\left(\pi^{\prime}\right)\right\}-\max \left\{L_{i}(\pi), L_{j}(\pi)\right\}<0\right\} \\
& \left.\quad \geq 2 \alpha \xi_{i}>\xi_{j}\right\}+M\left\{d_{i}>d_{j}\right\}-1 \\
& \quad \geq 2 .
\end{aligned}
$$

It implies that the optimal solution can be obtained by the EDD rule. The theorem is proved.

Note that these theorems have a higher dependence on the confidence level in the case of pessimistic value. For instance, the condition that the confidence level $\alpha$ is greater than 0.75 is sufficient for the result of Theorem 7. If the confidence level $\alpha$ is less than 0.75 , the required result may not be deduced based on the current method in the proof of Theorem 7. Also the confidence level $\alpha$ greater than 0.75 shows that the conclusion holds at a high confidence level. It is reasonable since we generally do not consider the situation that a result holds at a lower confidence level in reality. The pessimistic value case also shows that the decision maker is more inclined to believe that the rule can effectively obtain the optimal solution when faced with making decisions.

\section{Sensitivity Analysis}

Numerical examples are conducted to investigate the sensitivity of these confidence levels. Assume that there are 30 jobs; $\beta=2$; processing time of job $i$ is generated from uncertain normal distribution: $N(15,10)$ for $i=1,2, \ldots, 50$; due date of job $i$ is generated from uncertain normal distribution: $N(60,30)$ for $i=1,2, \ldots, 50 ; w_{i}=1.5 ; a$ obeys 


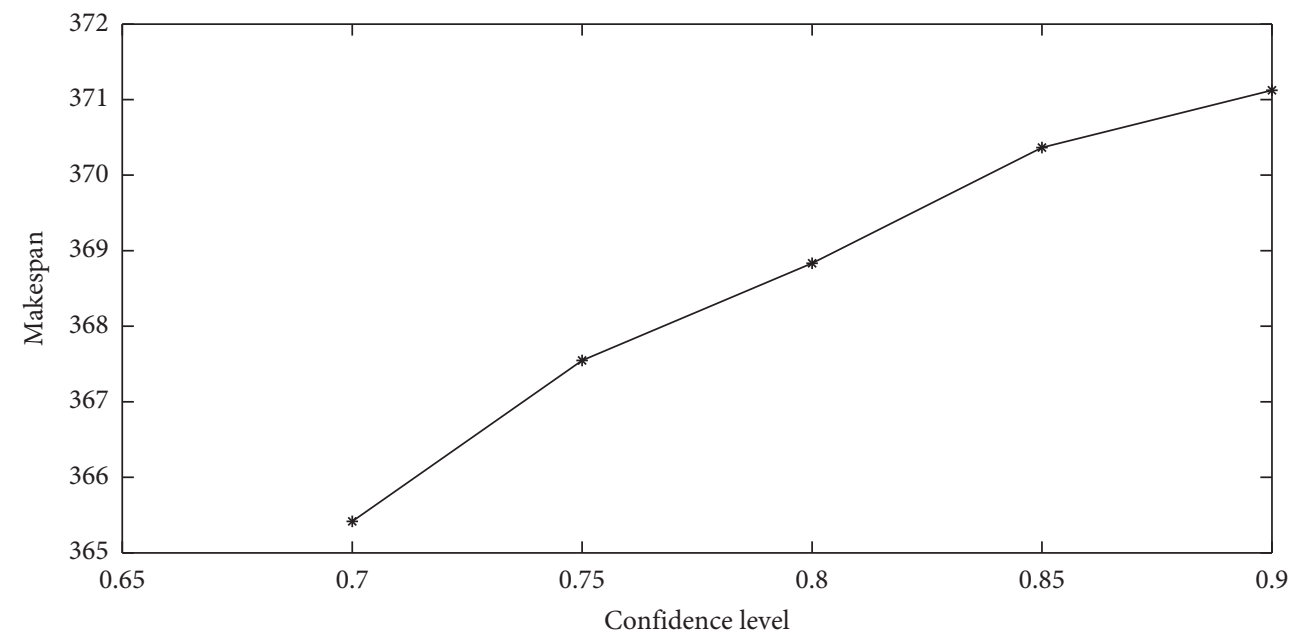

FIgURE 1: The sensitivity of solution for makespan problem.

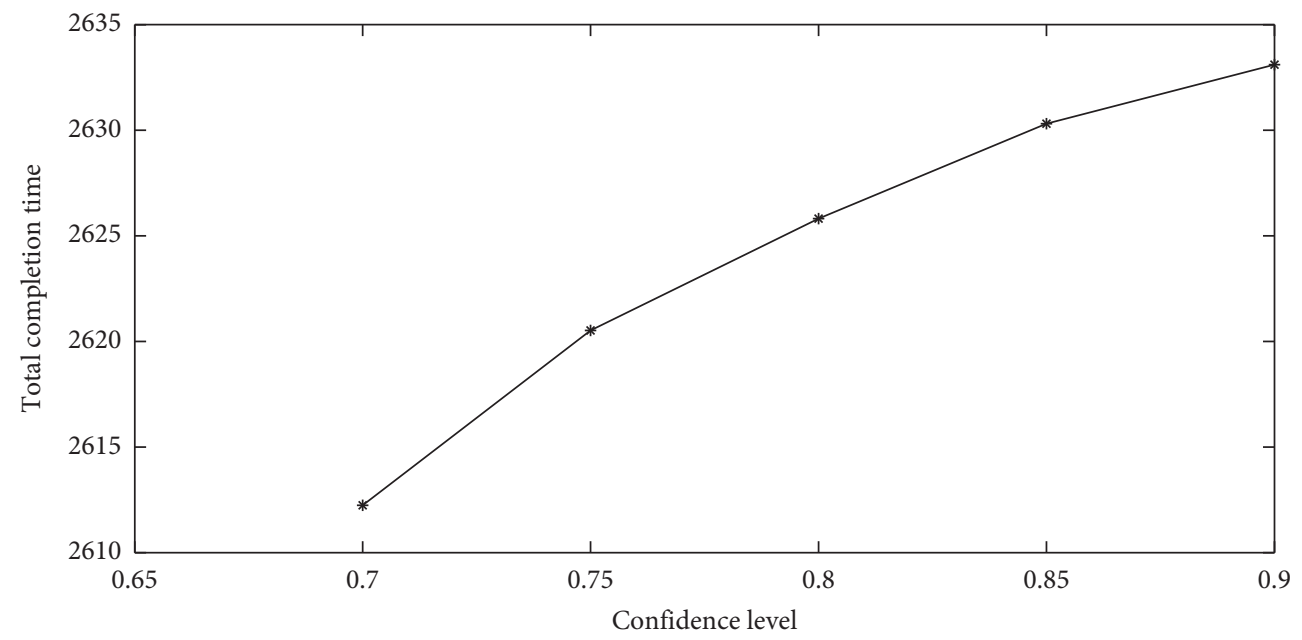

Figure 2: The sensitivity of solution for total completion time problem.

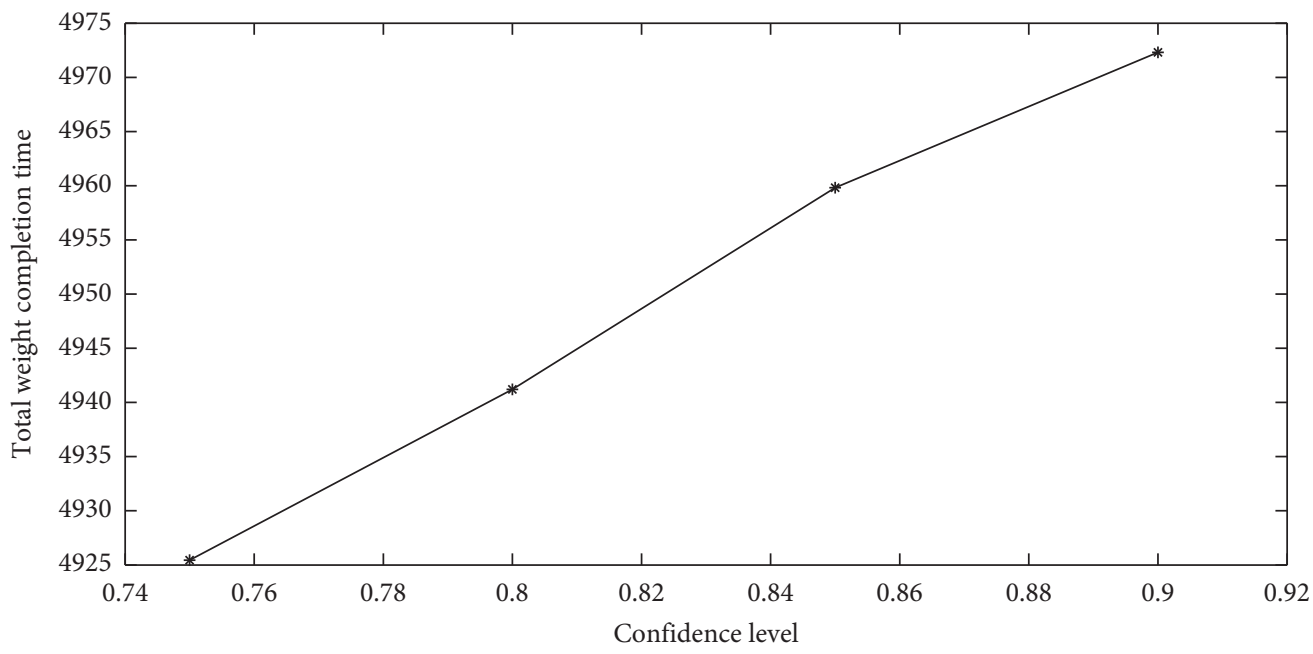

FIgURE 3: The sensitivity of solution for total weight completion time problem. 


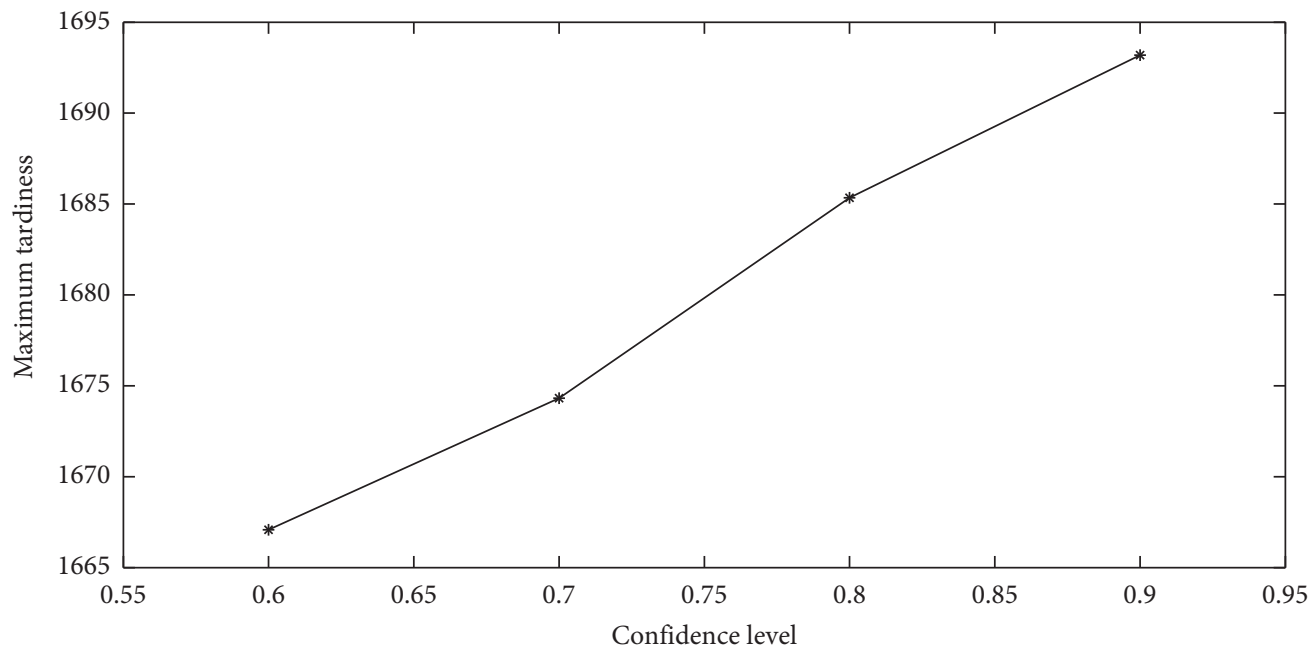

Figure 4: The sensitivity of solution for maximum lateness problem.

normal distribution $N(-0.2,0.1)$; and deterioration function $\alpha(t)=(t / 2)$. To examine the sensitivity of confidence levels $\alpha_{i}, i=1,2, \ldots, 4$, Figures $1-4$ indicate that the objective values are nondecreasing with respect to these confidence levels. It can be seen that these confidence levels must be greater than 0.6. Since the confidence level is too low, there is no guarantee that the conclusion of the theorem will be established in the case of pessimistic value.

\section{Conclusions}

An uncertain single-machine scheduling problem with deterioration and learning effect was studied in this paper. The processing time, due date, and learning coefficient were considered uncertain variables. Two different criteria (expected value and pessimistic value) multiobjective programming models were proposed. The equivalence of the two models was proved based on the uncertainty inverse distribution method. Due to the complexity of the problem, different dispatching rules was used to solve the problem. It was proved that the SPT rule can solve the makespan and total completion time problems under different uncertain criteria optimally, and the total weight completion time and maximum lateness can be solved by WSPT and EDD rules optimally, respectively. The results also suggested that the decision maker will choose the scheme with relatively high confidence level. At the last, the sensitivity analysis revealed that the objective function values are nondecreasing with respect to the confidence levels. For future work, it is an interesting research to investigate the influence of these uncertain variables on other workshop environment (e.g., parallel machine, flow shop, and job shop).

\section{Data Availability}

The data used to support the findings of this study are included within the article.

\section{Conflicts of Interest}

The author declares that there are no conflicts of interest regarding the publication of this paper.

\section{Acknowledgments}

This work was supported by the National Natural Science Foundation of China (no. 61673011) and Research Foundation of NIIT (YK18-10-02 and YK18-10-03).

\section{References}

[1] K. McKay, M. Pinedo, and S. Webster, "Practice-focused research issues for scheduling systems," Production \& Operations Management, vol. 11, pp. 249-258, 2002.

[2] J. Bai, Z.-R. Li, and X. Huang, "Single-machine group scheduling with general deterioration and learning effects," Applied Mathematical Modelling, vol. 36, no. 3, pp. 12671274, 2012.

[3] T. C. E. Cheng, S.-C. Tseng, P.-J. Lai, and W.-C. Lee, "Singlemachine scheduling with accelerating learning effects," Mathematical Problems in Engineering, vol. 2013, Article ID 816235, 7 pages, 2013.

[4] X. Huang, G. Li, Y. Huo, and P. Ji, "Single machine scheduling with general time-dependent deterioration, position-dependent learning and past-sequence-dependent setup times," Optimization Letters, vol. 7, no. 8, pp. 1793-1804, 2013.

[5] W.-H. Kuo and D.-L. Yang, "Single-machine scheduling problems with the time-dependent learning effect," Computers \& Mathematics with Applications, vol. 53, no. 11, pp. 1733-1739, 2007.

[6] W.-C. Lee, "Single-machine scheduling with past-sequencedependent setup times and general effects of deterioration and learning," Optimization Letters, vol. 8, no. 1, pp. 135-144, 2014.

[7] M. Toksarı, D. Oron, and E. Güner, "Single machine scheduling problems under the effects of nonlinear deterioration and time-dependent learning," Mathematical and Computer Modelling, vol. 50, no. 3-4, pp. 401-406, 2009.

[8] J.-B. Wang, "Single-machine scheduling problems with the effects of learning and deterioration," Omega, vol. 35, no. 4, pp. 397-402, 2007.

[9] X. Wang and T. C. Edwin Cheng, "Single-machine scheduling with deteriorating jobs and learning effects to minimize the makespan," European Journal of Operational Research, vol. 178, no. 1, pp. 57-70, 2007. 
[10] J.-B. Wang, Y. Jiang, and G. Wang, "Single-machine scheduling with past-sequence-dependent setup times and effects of deterioration and learning," The International Journal of Advanced Manufacturing Technology, vol. 41, no. 11-12, pp. 1221-1226, 2009.

[11] J.-B. Wang, "Single machine scheduling with a time-dependent learning effect and deteriorating jobs," Journal of the Operational Research Society, vol. 60, no. 4, pp. 583-586, 2009.

[12] J.-B. Wang and C. Wang, "Single-machine due-window assignment problem with learning effect and deteriorating jobs," Applied Mathematical Modelling, vol. 35, no. 8, pp. 4017-4022, 2011.

[13] L.-Y. Wang and E.-M. Feng, "A note on single-machine scheduling problems with the effects of deterioration and learning," The International Journal of Advanced Manufacturing Technology, vol. 59, no. 5-8, pp. 539-545, 2012.

[14] C.-C. Wu and W.-C. Lee, "Single-machine group-scheduling problems with deteriorating setup times and job-processing times," International Journal of Production Economics, vol. 115, no. 1, pp. 128-133, 2008.

[15] D.-L. Yang and W.-H. Kuo, "Single-machine scheduling with both deterioration and learning effects," Annals of Operations Research, vol. 172, no. 1, pp. 315-327, 2009.

[16] Y. Yin and D. Xu, "Some single-machine scheduling problems with general effects of learning and deterioration," Computers \& Mathematics with Applications, vol. 61, no. 1, pp. 100-108, 2011.

[17] X. Zhang and G. Yan, "Single-machine group scheduling problems with deteriorated and learning effect," Applied Mathematics and Computation, vol. 216, no. 4, pp. 1259-1266, 2010.

[18] Y. Zhang, X. Wu, and X. Zhou, "Stochastic scheduling problems with general position-based learning effects and stochastic breakdowns," Journal of Scheduling, vol. 16, no. 3, pp. 331-336, 2013.

[19] H. M. Soroush, "Stochastic bicriteria single machine scheduling with sequence-dependent job attributes and job-dependent learning effects," European Journal of Industrial Engineering, vol. 8, no. 4, pp. 421-456, 2014.

[20] H. Li, "Stochastic single-machine scheduling with learning effect," IEEE Transactions on Engineering Management, vol. 64, no. 1, pp. 94-102, 2017.

[21] F. Ahmadizar and L. Hosseini, "Single-machine scheduling with a position-based learning effect and fuzzy processing times," The International Journal of Advanced Manufacturing Technology, vol. 56, no. 5-8, pp. 693-698, 2011.

[22] F. Ahmadizar and L. Hosseini, "Minimizing makespan in a single-machine scheduling problem with a learning effect and fuzzy processing times," The International Journal of Advanced Manufacturing Technology, vol. 65, no. 1-4, pp. 581587, 2013.

[23] M. D. Toksari and O. A. Arık, "Single machine scheduling problems under position-dependent fuzzy learning effect with fuzzy processing times," Journal of Manufacturing Systems, vol. 45, pp. 159-179, 2017.

[24] B. Liu, Uncertainty Theory, Springer-Verlag, Berlin, Germany, 2nd edition, 2007.

[25] B. Liu, Uncertainty Theory: A Branch of Mathematics for Modeling Human Uncertainty, Springer-Verlag, Berlin, Germany, 2010.

[26] X. Chen and D. A. Ralescu, "Liu process and uncertain calculus," Journal of Uncertainty Analysis and Applications, vol. 1, no. 1, 2013.
[27] X. Chen, "Uncertain calculus with finite variation processes," Soft Computing, vol. 19, no. 10, pp. 2905-2912, 2015.

[28] K. Yao, "Multi-dimensional uncertain calculus with liu process," Journal of Uncertain Systems, vol. 8, no. 4, pp. 244-254, 2014.

[29] Z. Chen, Y. Lan, and R. Zhao, "Impacts of risk attitude and outside option on compensation contracts under different information structures," Fuzzy Optimization and Decision Making, vol. 17, no. 1, pp. 13-47, 2018.

[30] Y. Liu and D. A. Ralescu, "Value-at-risk in uncertain random risk analysis," Information Sciences, vol. 391-392, pp. 1-8, 2017.

[31] J. Zhou, Y. Liu, X. Zhang, X. Gu, and D. Wang, "Uncertain risk aversion," Journal of Intelligent Manufacturing, vol. 28, no. 3, pp. 615-624, 2017.

[32] X. Chen and Y. Ning, "The pth moment exponential stability of uncertain differential equation," Journal of Intelligent \& Fuzzy Systems, vol. 33, no. 2, pp. 725-732, 2017.

[33] Y. Feng, X. Yang, and G. Cheng, "Stability in mean for multidimensional uncertain differential equation," Soft Computing, vol. 22, no. 17, pp. 5783-5789, 2018.

[34] H. Liu and W. Fei, "Neutral uncertain delay differential equations," Information: An International Interdisciplinary Journal, vol. 16, no. 2, pp. 1225-1232, 2013.

[35] K. Yao, J. Gao, and Y. Gao, "Some stability theorems of uncertain differential equation," Fuzzy Optimization and Decision Making, vol. 12, no. 1, pp. 3-13, 2013.

[36] Q. Cui and Y. Sheng, "Uncertain programming model for solid transportation problem," Information: An International Interdisciplinary Journal, vol. 16, no. 2, pp. 1207-1214, 2013.

[37] R. Mu, Y. Lan, and W. Tang, "An uncertain contract model for rural migrant worker's employment problems," Fuzzy Optimization and Decision Making, vol. 12, no. 1, pp. 29-39, 2013.

[38] J. Shen and Y. Zhu, "Uncertain flexible flow shop scheduling problem subject to breakdowns," Journal of Intelligent \& Fuzzy Systems, vol. 32, no. 1, pp. 207-214, 2017.

[39] J. Shen, "An uncertain parallel machine problem with deterioration and learning effect," Computational and Applied Mathematics, vol. 38, no. 1, pp. 1-17, 2019.

[40] X. Zhang, L. Li, and G. Meng, "A modified uncertain entailment model," Journal of Intelligent \& Fuzzy Systems, vol. 27, no. 1, pp. 549-553, 2014.

[41] B. Liu, "Some research problems in uncertainty theory," Journal of Uncertain Systems, vol. 3, no. 1, pp. 3-10, 2009.

[42] W. Lee, "A note on deteriorating jobs and learning in singlemachine scheduling problems," International Journal of Business and Economics, vol. 3, pp. 83-89, 2004. 